$\Rightarrow$ VASCULAR DISEASE

\section{Treatment of ischaemic vascular disease with 3D-printed vessels}

Guideline-recommended approaches for revascularization in ischaemic vascular disease include artery bypass graft surgery and percutaneous intervention. The use of 3D-printed vascular patches might be a complementary strategy, according to new research published in Nature Biomedical Engineering.

Christopher Chen and colleagues used $3 \mathrm{D}$ printing to organize endothelial cells into patterned channels within a fibrin patch, and created vascular grafts containing endothelial-cell-lined lumens.

In a mouse model of hind-limb ischaemia (occlusion of the left femoral artery leading to a $50 \%$ postoperative decrease in perfusion of the distal ischaemic limb; image), implantation of vascular patches restored perfusion and rescued the muscle from ischaemic damage, such as capillary loss and muscle atrophy. The investigators observed direct anastomoses between the native and graft vasculature, producing Patterning of the vascular architecture was essential to restoration of perfusion; when endothelial cells

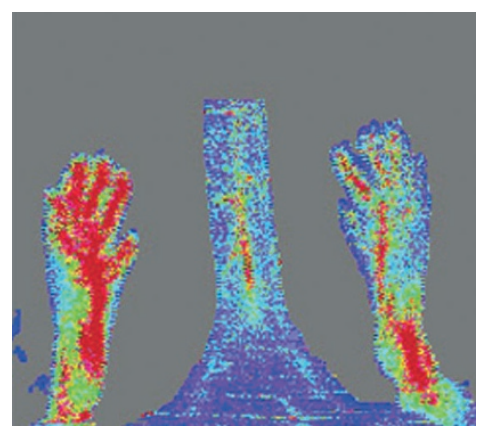

Reprinted from Mirabella, T. et al. 3D-printed vascular networks direct therapeutic angiogenesis in ischaemia. Nat. Biomed. Eng. 1, 0083 (2017) (C) Macmillan Publishers Limited a functional collateral circulation. were not organized into vessel-like structures, biointegration into a collateral circulation failed.

In a rat model of myocardial infarction (ligation of the left anterior descending coronary artery), Chen et al. placed vascular patches on the surface of the heart, spanning the ischaemic and nonischaemic zones. The patches were associated with increased vascularization throughout the implant and surrounding native tissue, and partial rescue of cardiac function.

"The findings suggest that the organization of cells within a patch guides the structural organization of vasculature formed by the host," comments Chen. "We are planning to test whether these $3 \mathrm{D}$-printing methods will continue to be effective when scaled to larger defects and disease models."

Gregory B. Lim

ORIGINAL ARTICLE Mirabella, T. et al. 3D-printed vascular networks direct therapeutic angiogenesis in ischaemia. Nat. Biomed. Eng. 1, 0083 (2017) FURTHER READING Giannopoulos, A. A. et al. Applications of 3D printing in cardiovascular diseases. Nat. Rev. Cardiol. 13, 701-718 (2016) 